\title{
Monolithic Acidic Catalysts for the Dehydration of Xylose into Furfural
}

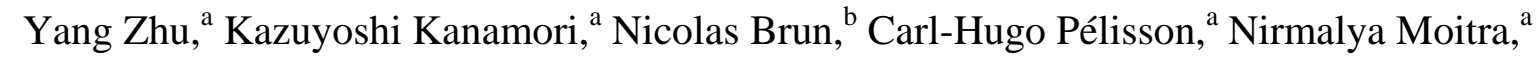
François Fajula, ${ }^{\mathrm{b}}$ Vasile Hulea, ${ }^{\text {b* }}$ Anne Galarneau, ${ }^{\mathrm{b}}$ Kazuyuki Takeda, ${ }^{\mathrm{a}}$ Kazuki Nakanishi ${ }^{\mathrm{a}^{*}}$

a Department of Chemistry, Graduate School of Science, Kyoto University, Kitashirakawa, Sakyo-ku, Kyoto 606-8502, Japan

${ }^{\mathrm{b}}$ Institut Charles Gerhardt Montpellier, UMR 5253 CNRS-UM-ENSCM, ENSCM, 8 rue de l'Ecole Normale, 34296 Montpellier Cedex 05, France

*Corresponding authors:

Professor Dr. Vasile Hulea, Institut Charles Gerhardt Montpellier, UMR 5253 CNRS-UM-ENSCM, ENSCM, 8 rue de l'Ecole Normale, 34296 Montpellier Cedex 05, France

E-mail: vasile.hulea@enscm.fr

TEL/FAX: +33 (0)467163464

Associate Professor Dr. Kazuki Nakanishi, Department of Chemistry, Graduate School of Science, Kyoto University, Kitashirakwa, Sakyo-ku, Kyoto 606-8502, Japan

E-mail: kazuki@kuchem.kyoto-u.ac.jp

TEL/FAX: +8175 7532925 
Keywords: zirconium phosphate, hierarchical porosity, monolith, xylose, dehydration

\begin{abstract}
We report the application of hierarchically porous zirconium phosphate monoliths with high surface area as acidic heterogeneous catalysts for the dehydration of xylose into furfural. Analyses by $\mathrm{NH}_{3}$-temperature programmed desorption and ${ }^{31} \mathrm{P}$ solid state NMR reveal the presence of both Lewis and Brønsted acid sites in the as-synthesized zirconium phosphate monolith and that calcined at $600{ }^{\circ} \mathrm{C}$. High accessibility and availability of the acidic sites and easy separation of the monolith from the liquid medium result in good catalytic activity (initial reaction rate for furfural production as $8.7 \mathrm{mmol}$ $\mathrm{g}_{\text {cat }}{ }^{-1} \mathrm{~h}^{-1}$ ) with easy handling of the catalyst.
\end{abstract}




\section{Introduction}

The reactivity and accessibility of active sites are two key factors for the efficiency of a reaction happening at a heterogeneous interface. The solution to the latter is to introduce a well-defined pore structure in heterogeneous catalysts, which not only ensures large interfacial area but also essentially minimizes the barrier from diffusion kinetics. Together with a satisfactory reactivity of the active sites, such materials with embedded optimized pore system commonly demonstrate catalytic behaviors as superior as those of homogeneous or enzymatic analogues. In this perspective, hierarchically porous monoliths may stand out from other material forms such as particles and thin films. The three-dimensionally interconnected macropores ( $d_{\text {pore }}>50 \mathrm{~nm}$ by IUPAC definition), typically in a few micrometer range, ensure efficient mass transfer over the whole substrate, especially when a pressure-driven flow is applied. Meanwhile, a network of mesopores (2 $\left.\mathrm{nm}<d_{\text {pore }}<50 \mathrm{~nm}\right)$ and micropores $\left(d_{\text {pore }}<2 \mathrm{~nm}\right)$ veined in the continuous macropore skeletons provides high surface area as well as short diffusion paths to the catalytic sites [1-5]. Moreover, hierarchically porous monoliths can be easily applied to a continuous flow set-up, which has been proven as a good candidate to replace conventional fixed-bed reactors [6-8].

Zirconium phosphate (ZrP) as a well-known and studied acidic catalyst has been extensively applied for catalytic reactions, such as dehydration [9-11], alkylation [12], acylation [13] and esterification [14] where acidic sites are required. The presence of both Lewis (exposed Zr sites) and Brønsted acidic sites (-P-OH) in ZrP materials makes them highly adaptable to different reactions and reacting systems. However, the introduction of hierarchically porous structure into such ZrP materials is somehow difficult due to its low solubility in aqueous medium, which commonly leads to crystalline precipitates with low surface area. We have recently succeeded in the synthesis of hierarchically porous ZrP monolith via sol-gel accompanied by phase separation starting from ionic precursors [15]. The micrometer-scale co-continuous macropores are formed as a result of phase separation (spinodal decomposition) during the sol-gel transition, while the nanometer-scale micro/mesopores derive from the interstitials between the constituent nanoparticles. 
The catalytic properties of the obtained hierarchically porous $\mathrm{ZrP}$ monolith were studied adopting the dehydration of xylose into furfural as a model reaction. Furfural, its analogues (2-methyl furan, 2.5-dimethylfuran, 5-(ethoxymethyl)furfural, etc.) and derivatives (tetrahydrofuran, levulinic acid, 2,5-furandicarboxylic acid, maleic acid, etc.) have been envisaged as platform chemicals for biofuel, biochemical and biopolymer industries [16,17]. Due to the high activation barrier for the reaction to be catalyzed by the Brønsted acid, high operating temperature and long residence time are often required in order to obtain a high xylose conversion [18,19]. The combination of both Brønsted acid sites and Lewis acid sites in the $\mathrm{ZrP}$ monoliths successfully suppresses the energy barrier for the activation of the reaction, leading to higher furfural yield at relatively lower temperature [20,21]. Furthermore the relatively slow kinetics allows the reaction to be conducted in a batch mode, making it easier to reveal more insights into the acidic nature of the obtained $\mathrm{ZrP}$ monolith.

\section{Results and Discussion}

\subsection{ZrP monolith synthesis}

As reported previously [15], hierarchically porous $\mathrm{ZrP}$ monoliths can be obtained via sol-gel process accompanied by phase separation under the presence of polyethylene oxide (PEO) and polyacrylamide (PAAm) as the phase separation inducers. A preferential distribution of PAAm in the gel phase via strong coordination between amide groups and $\mathrm{Zr}$ stabilizes the as-formed nano colloids of ZrP from further growing into bulky entities. Meanwhile, a preferential distribution of PEO in the solvent phase increases the compatibility between the PAAm-containing gel phase and the PEO-containing solvent phase, leading to a controlled phase separation process. After removal of the solvent, a macroporous co-continuous structure, typical of the spinodal decomposition phase separation, is obtained (Figure 1A). Beyond macropores, micro/mesopores, derived from the interstitials of the loosely packed ZrP nano colloids are also obtained, as shown by electron microscopies (Figure S1 and Figure S2). The presence of both micropores and mesopores is also supported by the $\mathrm{N}_{2}$ sorption isotherm shown in Figure 1B. A BET 
surface area of $600 \mathrm{~m}^{2} \mathrm{~g}^{-1}$ can be determined together with a relatively sharp mesopore size distribution ranging from $2 \mathrm{~nm}$ to $7 \mathrm{~nm}$ with an average mesopore size of $5 \mathrm{~nm}$ and micro/mesopore volume as $0.68 \mathrm{~cm}^{3} \mathrm{~g}^{-1}$.

Elemental analysis by EDS revealed a molar ratio between $\mathrm{Zr}$ and $\mathrm{P}$ of 1/2, in accordance with the molar ratio of both precursors added in the starting composition. Results of CHN elemental analyses confirm the presence of $7 \mathrm{wt} \%$ PAAm in the gel phase and $\mathrm{ZrP}$ is the dominant part of the gel. Results of ${ }^{31} \mathrm{P}$ DDMAS NMR show that phosphorous is mainly incorporated in the gel as $\mathrm{HPO}_{4}{ }^{2-}(-20.7 \mathrm{ppm})$ with small amounts of $\mathrm{PO}_{4}{ }^{3-}(-27.4 \mathrm{ppm})$ and $\mathrm{H}_{2} \mathrm{PO}_{4}{ }^{-}(-12.0 \mathrm{ppm})$ (Figure 1C). The main composition of as-synthesized $\mathrm{ZrP}$ monolith is therefore $\mathrm{Zr}\left(\mathrm{HPO}_{4}\right)_{2}$, which is compositionally in accordance with $\alpha$ - $\mathrm{ZrP}$ $\left(\mathrm{Zr}\left(\mathrm{HPO}_{4}\right)_{2} \cdot \mathrm{H}_{2} \mathrm{O}\right)$. XRD pattern further reveals the poorly crystalline nature of the monolith, which is commonly observed in $\mathrm{ZrP}$ materials synthesized at low temperature [22] (Figure 1D). The broad diffraction peak indexed at around $6^{\circ}$ indicates an expanded layered structure with the interlayer distance of $1.8 \mathrm{~nm}$.

Figure 1.

$\mathrm{NH}_{3}$-temperature programmed desorption (TPD) is commonly used for the determination of the surface acidity of heterogeneous acidic catalysts, due to the interaction between the acidic sites and ammonia molecules [23,24]. From the relationship between temperature and the desorption amount of adsorbed ammonia molecules, the relative amount of acidic sites can be detailed and their strength can be estimated. The constituent phase of the material should remain constant during the measurement at elevated temperatures in order to correctly measure the acidity of the material. Hence, due to the combustion of PAAm from the gel phase as well as the further dehydration of the ZrP network at elevated temperatures, the surface acidity of as-synthesized $\mathrm{ZrP}$ monolith cannot be measured with high enough accuracy. Instead, $\mathrm{ZrP}$ monolith calcined at $600{ }^{\circ} \mathrm{C}$ was used for the $\mathrm{NH}_{3}$-TPD measurement in order to obtain some information, though indirect, about the initial surface acidity of the as-synthesized $\mathrm{ZrP}$ monolith (Figure 2). Significant desorption of ammonia starts from $200{ }^{\circ} \mathrm{C}$ and completes at $500{ }^{\circ} \mathrm{C}$, during which a broad peak with a maximum rate of desorption at around $300{ }^{\circ} \mathrm{C}$ together with a small shoulder at around $400{ }^{\circ} \mathrm{C}$ is observed. The results indicate that the main acidic sites in the monolith are of medium 
strength $\left(0.75 \mathrm{mmol} \mathrm{g}{ }^{-1}\right.$, desorbing ammonia from $200{ }^{\circ} \mathrm{C}$ to $\left.400{ }^{\circ} \mathrm{C}\right)$ together with the presence of a fraction of strong acidic sites $\left(0.08 \mathrm{mmol} \mathrm{g}^{-1}\right.$, desorbing ammonia from $400{ }^{\circ} \mathrm{C}$ to $\left.500{ }^{\circ} \mathrm{C}\right)$ [25]. The total amount of acidic sites $\left(0.83 \mathrm{mmol} \mathrm{g}^{-1}\right)$ as well as the strength distribution is comparable with that of the well-known ZSM-5 zeolite ( $\mathrm{Si} / \mathrm{Al}=15$, $0.86 \mathrm{mmol} \mathrm{g}^{-1}$ in total amount, $0.09 \mathrm{mmol} \mathrm{g}^{-1}$ as weak acidic sites $\left(<200{ }^{\circ} \mathrm{C}\right), 0.68 \mathrm{mmol}$ $\mathrm{g}^{-1}$ as medium strong acidic sites $\left(200-400{ }^{\circ} \mathrm{C}\right)$ and $0.09 \mathrm{mmol} \mathrm{g}^{-1}$ as strong acidic sites (400-500 ${ }^{\circ} \mathrm{C}$ ), Figure 2). According to the ${ }^{31} \mathrm{P}$ DDMAS NMR spectrum of ZrP monolith calcined at $600{ }^{\circ} \mathrm{C}$ (Figure S3), the main phosphorous species incorporated in the monolith changes from $\mathrm{HPO}_{4}{ }^{2-}$ to $\mathrm{PO}_{4}{ }^{3-}$ (-30.5 ppm), indicating a sharp decrease of the amount of Brønsted acid sites [26]. However, a higher number of free $\mathrm{Zr}$ sites as the accessible Lewis acid sites are deduced to be generated due to the removal of PAAm that coordinates with the $\mathrm{Zr}$ sites. Therefore it can be further speculated that there are more Brønsted acid sites and less Lewis acid sites in as-synthesized ZrP monolith. The BET surface area and micro/mesopore volume decrease after calcination $\left(340 \mathrm{~m}^{2} \mathrm{~g}^{-1}\right.$ and $0.34 \mathrm{~mL} \mathrm{~g}^{-1}$ versus 600 $\mathrm{m}^{2} \mathrm{~g}^{-1}$ and $0.68 \mathrm{~mL} \mathrm{~g}^{-1}$ before calcination), while the mesopore size $(5 \mathrm{~nm})$ remains similar to the one obtained for the as-synthesized ZrP monolith (Figure S4 inset).

\section{Figure 2.}

\subsection{Catalytic performances}

Dehydration of xylose into furfural was investigated in batch mode (Table 1). Without the addition of catalyst, only $5 \%$ xylose conversion was obtained with $80 \%$ selectivity into furfural (Entry 1). The conversion increased to $18 \%$ with $50 \%$ furfural selectivity using the as-synthesized ZrP monolith as a catalyst under the same reaction conditions (Entry 2). The lower furfural selectivity in the catalytic process is due to the side catalyzed reactions, involving both furfural and xylose. The activity of the $\mathrm{ZrP}$ monolith calcined at $600{ }^{\circ} \mathrm{C}$ was also evaluated (Entry 4). Though the conversion and furfural selectivity are lower than those of the as-synthesized $\mathrm{ZrP}$ monolith, the amount of xylose converted on unit surface area of calcined $\mathrm{ZrP}$ monolith was higher.

Such result can be explained by a compensation effect resulting from a change of the nature of the acid sites and a decrease of the amount of active surface in the monolith after 
the calcination. As reported recently [9], the acid-catalyzed xylose dehydration to furfural involves the reversible isomerization of xylose to xylulose and lyxose catalyzed by Lewis acid sites and the irreversible dehydration of the isomers to furfural catalyzed by Brønsted acid sites. The activation energies for both processes are 15.5 and $23.1 \mathrm{kcal} \mathrm{mol}^{-1}$, respectively. A higher activation energy of $32 \mathrm{kcal} \mathrm{mol}^{-1}$ is observed when xylose is directly converted to furfural catalyzed by Brønsted acid sites. As abovementioned, more $\mathrm{Zr}$ Lewis acid sites are exposed after the calcination at $600{ }^{\circ} \mathrm{C}$, making easier the isomerization reaction to proceed, and hence significantly lowering the overall activation energy required for the system. However, due to the simultaneously decrease of the number of Brønsted acid sites, the amount of isomers that can be further converted to furfural was limited. On the other hand, though the amount of Brønsted acid sites in the as-synthesized $\mathrm{ZrP}$ monolith is higher, the overall activation energy for the system is still higher than that of calcined ZrP monolith due to the smaller amount of Lewis acid sites. Nevertheless, due to the higher surface area of as-synthesized $\mathrm{ZrP}$ monolith, the overall catalytic activity is still higher than that of the calcined monolith. Hence, in this research, the as-synthesized ZrP monolith was chosen as the standard catalyst.

Although the xylose conversion and furfural yield increased with the reaction temperature (Entry 2, 8, 9), the increase in selectivity of furfural was relatively minor: from $50 \%$ to 62 $\%$ up to $160{ }^{\circ} \mathrm{C}$. A further increase of the temperature to $170{ }^{\circ} \mathrm{C}$, however, slightly decreased the selectivity to $59 \%$. The extension of reaction time improved the conversion of xylose to furfural together with an increased formation of the insoluble brownish polymeric byproduct, humins, while the selectivity remained approximately the same. This result indicates that the reactivity of the catalyst is stable against the formation of byproducts. Such behavior is quite unique during the catalytic dehydration of lignocellulosic products over porous catalysts as it is usually found that the activity of the catalyst rapidly decreases due to the formation of byproducts such as coke and humins, which block micropores and small mesopores and reduces the accessibility of the feed molecules to the catalytic sites in the catalyst [27]. A decrease in the selectivity towards furfural was observed when nearly total conversion of xylose (97\%) was obtained at $170{ }^{\circ} \mathrm{C}$ for $24 \mathrm{~h}$. 
Amorphous particles of $\mathrm{ZrP}$ have been reported as catalysts for xylose dehydration, showing $56 \%$ xylose conversion and $38 \%$ furfural selectivity after reaction at $160{ }^{\circ} \mathrm{C}$ for 4 $\mathrm{h}$ with microwave heating under stirring [20]. In comparison, our hierarchically porous ZrP monolith shows a higher furfural selectivity (62 \%) and slightly lower xylose conversion (42\%) at the same temperature and reaction time without the aid of microwave heating or stirring to accelerate the diffusion of the guest molecules. Recently, Cheng et al. reported $96 \%$ xylose conversion and $54 \%$ furfural selectivity by using amorphous ZP particles as the catalyst at $170{ }^{\circ} \mathrm{C}$ for $2 \mathrm{~h}$ in aqueous medium with xylose/catalyst weight ratio of $4 / 1$ [28]. Although the results we have obtained at the same temperature for $4 \mathrm{~h}$ (59 \% xylose conversion, $59 \%$ furfural selectivity) were modest, much less catalyst (xylose/catalyst weight ratio of 15/1) was used in our experiment. Consequently, the obtained reaction rate of $8.7 \mathrm{mmol} \mathrm{g}_{\mathrm{cat}}{ }^{-1} \mathrm{~h}^{-1}$ for the as-synthesized ZrP monolith at $170{ }^{\circ} \mathrm{C}$, though not optimized ( batch mode, no stirring), results to be higher than that reported when using ZrP particles (6.9 mmol $\mathrm{g}_{\text {cat }}^{-1} \mathrm{~h}^{-1}$ ). In the meantime, in terms of reaction rate, $\mathrm{ZrP}$ monolith catalyst proves also superior to several other reported catalysts such as vanadyl pyrophosphate (1.3 mmol g ${ }_{c a t}^{-1} \mathrm{~h}^{-1}$ ) [10], aluminosilicate (H-MCM-22, $2.4 \mathrm{mmol}_{\mathrm{cat}}{ }^{-1} \mathrm{~h}^{-1}$ ) [29], and silicoaluminophosphate (SAPO-5, $1.2 \mathrm{mmol} \mathrm{g}_{\mathrm{cat}}^{-1} \mathrm{~h}^{-1}$ ) [30]. ZrP monolith may constitute therefore an advantageous and promising material for the dehydration of xylose to furfural. The reaction at $150{ }^{\circ} \mathrm{C}$ for $4 \mathrm{~h}$ (Table 1, Entry 2) was repeated to check the reusability of the catalyst. Furfural selectivity is slightly increased (Table 1, Entry 3), probably because in the first stage of the process, some catalytic sites, responsible for the side reactions, are deactivated. This may also explain the higher selectivity obtained at longer reaction times (Entries 5, 6, 7). Note that in this research, water was used as a "greener" solvent than other frequently used solvents such as dimethyl sulfoxide, toluene or ionic liquid [31-33], and comparable performances are demonstrated. Together with the possibility to design continuous flow set-ups ${ }^{1}$, hierarchically porous $\mathrm{ZrP}$ monoliths demonstrate great potential as a sustainable catalyst for the dehydration reactions of lignocellulosic materials.

Table 1. 


\section{Conclusions}

A hierarchically porous ZrP monolith with high BET surface area of $600 \mathrm{~m}^{2} \mathrm{~g}^{-1}$ and micro/mesopore volume of $0.68 \mathrm{~cm}^{3} \mathrm{~g}^{-1}$ has been synthesized via a sol-gel process accompanied by phase separation starting from ionic precursors. The ZrP monolith calcined at $600{ }^{\circ} \mathrm{C}$ shows a large fraction of medium strong acidic sites and a small fraction of strong acidic sites, while the calcination at $600{ }^{\circ} \mathrm{C}$ leads to the decrease of Brønsted acid sites and increase of Lewis acid sites as compared with the as-synthesized ZrP monolith. The ZrP monoliths have been used for the catalytic dehydration of xylose into furfural. High activity and selectivity towards furfural were achieved at $170{ }^{\circ} \mathrm{C}$ with the as-synthesized ZrP catalyst.

The authors declare no competing financial interest.

\section{Acknowledgement}

The present work was financially supported by the Advanced Low Carbon Technology Research and Development Program (ALCA) from the Japan Science and Technology Agency (JST) and JSPS KAKENHI 15J00156.

\section{References}

[1] C. M. A. Parlett, K. Wilson, A. F. Lee, Chem. Soc. Rev. 42 (2013) 3876-3893.

[2] Z. Yuan, B. Su, J. Mater. Chem. 16 (2006) 663-677.

[3] A. Feinle, M. S. Elsaesser, N. Hüsing, Chem. Soc. Rev. 2016, DOI :

10.1039/c5cs00710k.

[4] K. Kanamori, K. Nakanishi, Chem. Soc. Rev. 40 (2011) 754-770.

[5] J.-H., Smått, C. Weidenthaler, J. B. Rosenholm, M. Lindén, Chem. Mater. 18 (2006) 1443-1450.

[6] A. E. Kadib, R. Chimenton, A. Sachse, F. Fajula, A. Galarneau, B. Coq, Angew. Chem. Int. Ed. 48 (2009) 4969-4972. 
[7] N. Moitra, K. Kanamori, Y. H. Ikuhara, X. Gao, G. Hasegawa, K. Takeda, Y. Zhu, T. Shimada, K. Nakanishi, J. Mater. Chem. A 2 (2014) 12535-12544.

[8] A. Cybulski, J. A. Moulijn, Catal. Rev. Sci. Eng. 36 (1994) 179-270.

[9] R. Weingarten, Y. T. Kim, G. A. Tompsett, A. Fernández, K. S. Han, E. W. Hagaman, W. C. Conner Jr., J. A. Dumesic, G. W. Huber, J. Catal. 304 (2013) 123-134.

[10] I. Sádaba, S. Lima, A. A. Valente, M. L. Granados, Carbohydr. Res. 346 (2011) 2785-2791.

[11] F. S. Asghari, H. Yoshida, Carbohydr. Res. 341 (2006) 2379-2387.

[12] A. R. Hajipour, H. Karimi, Appl. Catal. A: General 482 (2014) 99-107.

[13] F. Zhang, Y. Xie, W. Lu, X. Wang, S. Xu, X. Lei, J. Colloid Interf. Sci. 349 (2010) 571-577.

[14] K. N. Rao, A. Sridhar, A. F. Lee, S. J. Taverner, N. A. Young, K. Wilson, Green Chem. 8 (2006) 790-797.

[15] Y. Zhu, T. Shimizu, T. Kitashima, K. Morisato, N. Moitra, N. Brun, K. Kanamori, K. Takeda, M. Tafu, K. Nakanishi, New J. Chem. 39 (2015) 2444-2450.

[16] C. M. Lew, N. Rajabbeigi, M. Tsapatsis, Ind. Eng. Chem. Res. 51 (2012) 5364-5366.

[17] M. Mascal, E. B. Nikitin, ChemSusChem 2 (2009) 423-426.

[18] R. Weingarten, J. Cho, W. C. Conner Jr., G. W. Huber, Green Chem. 12 (2010)

1423-1429.

[19] M. J. Climent, A. Corma, S. Iborra, Chem. Rev. 111 (2011) 1072-1133.

[20] R. Weingarten, G. A. Tompsett, W. C. Conner Jr., G. W. Huber, J. Catal. 279 (2011) 174-182.

[21] V. Choudhary, S. I. Sandler, D. G. Vlachos, ACS Catal. 2 (2012) 2022-2028.

[22] A. Clearfield, J. A. Stynes, J. Inorg. Nucl. Chem. 26 (1964) 117-129.

[23] G. V. A. Martins, G. Berlier, C. Bisio, S. Coluccia, H. O. Pastore, L. Marchese, J. Phys. Chem. C 112 (2008) 7193-7200.

[24] M. Niwa, N. Katada, Chem. Rec. 13 (2013) 432-455.

[25] A. R. Hajipour, H. Karimi, Appl. Catal. A: General 482 (2014) 99-107.

[26] G. Alberti, M. Casciola, F. Marmottini, R. Vivani, J. Porous Mater. 6 (1999) 299-305.

[27] S. Lima, M. Pillinger, A. A. Valente, Catal. Commun. 9 (2008) 2144-2148.

[28] L. Y. Cheng, X. K. Guo, C. H. Song, G. Y. Yu, Y. M. Cui, N. H. Xue, L. M. Peng, X. F. Guo, W. P. Ding, RSC Adv. 3 (2013) 23228-23235.

[29] M. M. Antunes, S. Lima, A. Fernandes, M. Pillinger, M. F. Ribeiro, A. A. Valente, Appl. Catal. A: General 417-418 (2012) 243-252.

[30] S. Lima, A. Fernandes, M. M. Antunes, M. Pillinger, F. Ribeiro, A. A. Valente, Catal. Lett. 135 (2010) 41-47.

[31] E. Lam, E. Majid, A. C. W. Leung, J. H. Chong, K. A. Mahmoud, H. T. Luong, ChemSusChem 4 (2011) 535-541.

[32] B. Saha, M. M. Abu-Omar, Green Chem. 16 (2014) 24-38.

[33] H. B. Zhao, J. E. Holladay, H. Brown, Z. C. Zhang, Science 316 (2007) 1597-1600. 


\section{Figure caption}

Figure 1 (A) SEM image, (B) $\mathrm{N}_{2}$ adsorption-desorption isotherm (inset is the BJH pore size distribution obtained from the adsorption branch), (C) ${ }^{31} \mathrm{P}$ DDMAS NMR spectrum and (D) XRD pattern of the as-synthesized ZrP monolith.

Figure $2 \mathrm{NH}_{3}$-TPD curves of $\mathrm{ZrP}$ monolith calcined at $600{ }^{\circ} \mathrm{C}$ and $\mathrm{ZSM}-5$ as the benchmark.

Table 1 Catalytic activity of ZrP monoliths in the dehydration of xylose to furfural. 
Table 1 Catalytic activity of ZrP monoliths in the dehydration of xylose to furfural.

\begin{tabular}{ccccccc}
\hline Entry & Catal. & $\begin{array}{c}\text { Temp. } \\
/{ }^{\circ} \mathrm{C}\end{array}$ & $\begin{array}{c}\text { Reaction time / } \\
\mathrm{h}\end{array}$ & $\begin{array}{c}\text { Xylose conversion / } \\
\%\end{array}$ & $\begin{array}{c}\text { Furfural yield / } \\
\%\end{array}$ & $\begin{array}{c}\text { Selectivity } \\
/ \%\end{array}$ \\
\hline 1 & None & 150 & 4 & 5 & 4 & 80 \\
2 & $\mathrm{ZrP}$ & 150 & 4 & 18 & 9 & 50 \\
3 & $\mathrm{ZrP}-\mathrm{R} 2^{*}$ & 150 & 4 & 18 & 12 & 67 \\
4 & $\mathrm{ZrP} 600{ }^{\circ} \mathrm{C}$ & 150 & 4 & 13 & 6 & 46 \\
5 & $\mathrm{ZrP}$ & 150 & 8 & 32 & 18 & 56 \\
6 & $\mathrm{ZrP}$ & 150 & 16 & 50 & 28 & 56 \\
7 & $\mathrm{ZrP}$ & 150 & 24 & 67 & 38 & 57 \\
8 & $\mathrm{ZrP}$ & 160 & 4 & 42 & 26 & 62 \\
9 & $\mathrm{ZrP}$ & 170 & 4 & 59 & 35 & 59 \\
10 & $\mathrm{ZrP}$ & 170 & 24 & 97 & 47 & 49 \\
$*$
\end{tabular}



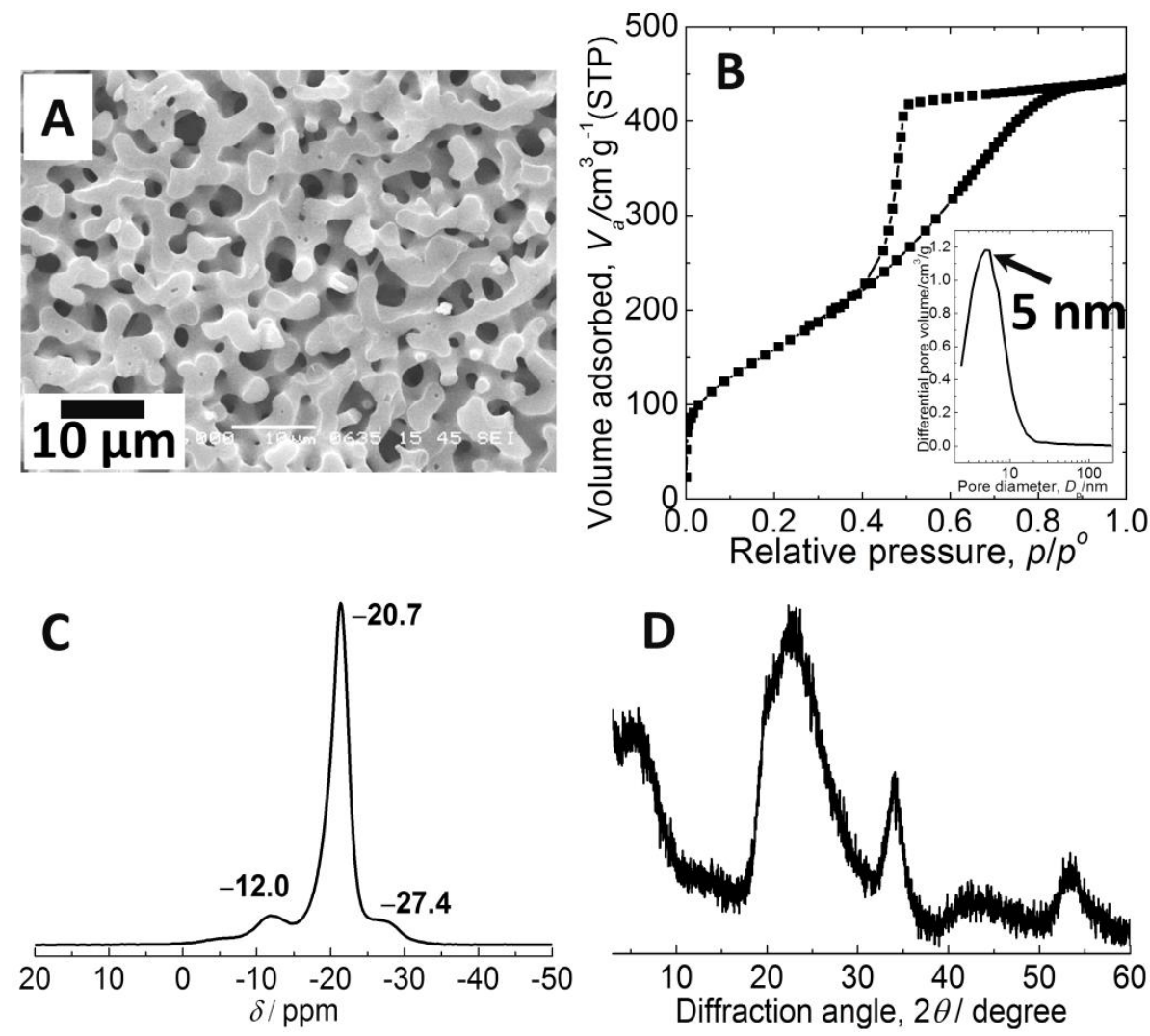

Figure 1 (A) SEM image, (B) $\mathrm{N}_{2}$ adsorption-desorption isotherm (inset is the BJH pore size distribution obtained from the adsorption branch), (C) ${ }^{31}$ P DDMAS NMR spectrum and (D) XRD pattern of the as-synthesized ZrP monolith. 


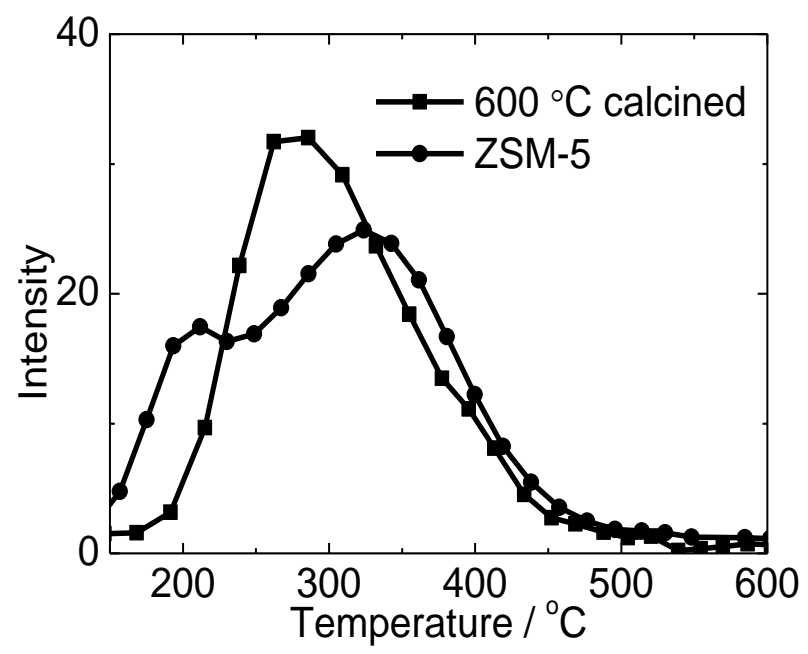

Figure $2 \mathrm{NH}_{3}$-TPD curves of $\mathrm{ZrP}$ monolith calcined at $600{ }^{\circ} \mathrm{C}$ and $\mathrm{ZSM}-5$ as the benchmark. 
